\title{
Crescimento e desenvolvimento de plantas jovens de açaizeiro sob déficit hídrico em
}

\section{Latossolo Amarelo}

\author{
Growth and development of young açai plants under water deficit in Oxisol \\ Crecimiento y desarrollo de plantas jóvenes de palma de açaí bajo déficit hídrico en un Oxisol
}

Amarillo

Recebido: 14/09/2021 | Revisado: 20/09/2021 | Aceito: 27/09/2021 | Publicado: 28/09/2021

Maria Soraia Fortado Vera Cruz

ORCID: https://orcid.org/0000-0003-2039-4644 Universidade Estadual do Oeste do Paraná, Brasil E-mail: soraiaf12@hotmail.com

Paula Martinelly dos Reis Farias

ORCID: https://orcid.org/0000-0002-1333-8858 Universidade Federal Rural da Amazônia, Brasil

E-mail: paulamartinelle@ hotmail.com

José Darlon Nascimento Alves

ORCID: https://orcid.org/0000-0003-1290-5598 Universidade Federal Rural da Amazônia, Brasil E-mail: jose.darllon@hotmail.com

Heráclito Eugênio Oliveira da Conceição ORCID: https://orcid.org/0000-0002-5193-0916 Universidade Federal Rural da Amazônia, Brasil E-mail: agroheraclito@yahoo.com.br Michel Sauma Filho ORCID: https://orcid.org/0000-0002-6603-8445 Universidade Federal Rural da Amazônia, Brasil E-mail: michel.sauma@ufra.edu.br João Vitor Silva e Silva ORCID: https://orcid.org/0000-0002-4673-8014 Universidade Federal Rural da Amazônia, Brasil E-mail: joaovitorsmg0716@gmail.com

Antônia Caroline Siqueira Aguiar ORCID: https://orcid.org/0000-0002-7634-7110 Universidade Federal Rural da Amazônia, Brasil E-mail: carolineaguiarmv@gmail.com

Priscila Martins da Silva ORCID: https://orcid.org/0000-0002-5129-282X Universidade Federal Rural da Amazônia, Brasil E-mail: martins.pri04@gmail.com

Madison da Costa Pinheiro ORCID: https://orcid.org/0000-0001-5537-4825 Universidade Federal Rural da Amazônia, Brasil E-mail: madisoncostta@gmail.com

Jairo Neves de Oliveira

ORCID: https://orcid.org/0000-0002-6415-8101 Universidade Federal Rural da Amazônia, Brasil E-mail: jairoufracap22@gmail.com

\begin{abstract}
Resumo
O açaizeiro é uma palmeira nativa da Amazônia. Apesar do potencial econômico apresentado atualmente por essa espécie, ainda existem poucos estudos relacionados ao desenvolvimento dessa planta frente a déficits hídricos. Este trabalho objetivou avaliar o crescimento e o desenvolvimento de plantas jovens de açaizeiro em Latossolo Amarelo sob diferentes níveis de estresse hídrico. O experimento foi desenvolvido em estufa agrícola localizada na Universidade Federal Rural da Amazônia, Campus de Capitão Poço. Os tratamentos foram dispostos em delineamento inteiramente casualizado com os seguintes tratamentos: irrigação diária com umidade do solo mantida entre 80 a $85 \%$ da capacidade de campo (testemunha); suspensão da irrigação durante 3 dias; 7 dias; 14 dias e 21 dias. Foram avaliadas a altura da planta, diâmetro do caule, número de folhas e folíolos, área foliar, comprimento e volume da raiz, conteúdo relativo de água, massas secas e alocações da biomassa em diferentes partes vegetativas. O Intervalo de irrigação de 21 dias proprocionou menores respostas das mudas para a maioria das variáveis analisadas. O déficit
\end{abstract}


hídrico ocasionou maior acúmulo massa seca da raiz e superior alocação da biomassa na raiz. Assim, a restrição hídrica influenciou negativamente o desenvolvimento das mudas de açaizeiro.

Palavras-chave: Estresse hídrico; Crescimento vegetativo; Euterpe oleracea Mart.

\begin{abstract}
The açai is a palm tree native to the Amazon. Despite the economic potential currently presented by this species, there are still few studies related to the development of this plant in the face of water deficits. This work aimed to evaluate the growth and development of young açaí palm plants in an Oxisol under different water stress levels. The experiment was carried out in an greenhouse located at the Federal Rural University of Amazônia, Campus de Capitão Poço. The treatments were arranged in a completely randomized design with the following treatments: daily irrigation with soil moisture maintained between 80 to $85 \%$ of field capacity (control); suspension of irrigation for 3 days; 7 days; 14 days and 21 days. Plant height, stem diameter, number of leaves and leaflets, leaf area, root length and volume, relative water content, dry mass and biomass allocations in different vegetative parts were evaluated. The 21 day irrigation interval provided lower seedling responses for most of the analyzed variables. The water deficit caused higher accumulation of root dry mass and higher allocation of biomass in the root. Thus, the water deficit negatively influenced the development of açaí palm seedlings.
\end{abstract}

Keywords: Water stress; Vegetative growth; Euterpe oleracea Mart.

\title{
Resumen
}

Açaí es una palmera nativa del Amazonas. A pesar del potencial económico que presenta actualmente esta especie, aún existen pocos estudios relacionados con el desarrollo de esta planta ante los déficits hídricos. Este trabajo tuvo como objetivo evaluar el crecimiento y desarrollo de plantas jóvenes de palma de açaí en un Oxisol bajo diferentes niveles de estrés hídrico. El experimento se llevó a cabo en un invernadero agrícola ubicado en la Universidad Federal Rural de la Amazonia, Campus Capitão Poço. Los tratamientos se dispusieron en un diseño completamente al azar con los siguientes tratamientos: riego diario con humedad del suelo mantenida entre 80 a $85 \%$ de la capacidad de campo (control); suspensión del riego durante 3 días; 7 días; 14 días y 21 días. Se evaluó la altura de la planta, el diámetro del tallo, el número de hojas y folíolos, el área foliar, la longitud y volumen de las raíces, el contenido relativo de agua, la masa seca y las asignaciones de biomasa en diferentes partes vegetativas. El intervalo de riego de 21 días proporcionó respuestas más bajas de las plántulas para la mayoría de las variables analizadas. El déficit hídrico provocó una mayor acumulación de masa seca radicular y una mayor asignación de biomasa en la raíz. Así, el déficit hídrico influyó negativamente en el desarrollo de plántulas de açaí.

Palabras clave: Estrés hídrico; Crecimiento vegetativo, Euterpe oleracea Mart.

\section{Introdução}

O açaizeiro (Euterpe oleracea Mart.) é uma palmeira nativa da Amazônia que ocorre em grandes extensões no estuário amazônico, sua importância econômica está centralizada na produção de frutos e palmito (Silvestre et al., 2016; Cordeiro et al., 2017; Silva et al., 2017; Araújo et al., 2018). O açaizeiro tem como principal centro de dispersão natural o Estado do Pará (Homma, 2006). É a palmeira mais produtiva do ecossistema amazônico. O Pará é o principal produtor de açaí do Brasil, representando 94\% da produção total em 2019 (1.398.328 toneladas) (IBGE, 2021). Nos últimos anos vem ocorrendo o aumento do cultivo do açaizeiro em terra firme, em que diferentes estudos estão sendo realizados para aprimorar o sistema produtivo, principalmente na fase de mudas, etapa crucial para o sucesso do cultivo em campo.

Um dos manejos essenciais na fase de mudas de açaizeiro é a disponibilidade adequada de da água. Em situações de déficits hídricos, há uma redução do desenvolvimento das plantas, redução na produção de folhas, diminuição na taxa fotossintética e menor acúmulo de biomassa (Taiz et al., 2017; Kerbauy, 2019). Isso acarreta no maior tempo na fase de viveiro e redução da uniformidade e sobrevivência das mudas em campo frente às diferentes condições ambientais. Porém, apesar da grande divulgação em nível nacional e internacional e, do potencial econômico apresentado atualmente por essa espécie, ainda existem poucos estudos com enfoque na determinação da demanda de água; do intervalo de irrigação ideal e a tolerância dessa cultura frente a déficits hídricos (Mar et al., 2013; Silvestre et al., 2016; 2017).

Estudos anteriores evidenciaram que o déficit hídrico ocasionou redução da produção de biomassa em mudas de açaizeiro (Mar et al., 2013) e que o déficit hídrico por dezesseis dias resultou em alterações expressivas nas variáveis bioquímicas avaliadas em plantas jovens de açaizeiro, principalmente nos teores de sacarose, carboidratos solúveis totais, 
prolina e glicina-betaína (Cordeiro et al, 2017). Apesar desses resultados, novos estudos são essenciais para melhor compreensão da tolerância do açaizeiro em diferentes condições edafoclimáticas na região amazônica, visto que o sistema de produção dessa cultura em campo ainda está sendo desenvolvido. Assim, novas pesquisas voltadas a essa cultura, ajudam para a maior produtividade da espécie, possibilitando maior retorno financeiro pelos agricultores.

Assim, o objetivo do trabalho foi avaliar a influência do déficit hídrico no crescimento e desenvolvimento de plantas jovens de açaizeiro (Euterpe oleracea Mart.).

\section{Metodologia}

O experimento foi desenvolvido em uma estufa agrícola localizada na Universidade Federal Rural da Amazônia, Campus de Capitão Poço, Capitão Poço, Pará, cujas coordenadas geográficas são: Latitude 1 44' 47" Sul e Longitude 47 $3^{\prime}$ 57" Oeste. O experimento teve início em 17 de dezembro de 2016 e foi finalizada em 28 de janeiro de 2017. Foram utilizadas mudas com seis meses de idade. O solo do local é caracterizado como Latossolo Amarelo textura média (EMBRAPA, 2013). O solo utilizado foi retirado a uma profundidade de $0,20 \mathrm{~m}$ de uma área de capoeira de aproximadamente 30 anos, distante 13 km da área experimental.

Na Figura 1 são apresentados os dados de temperaturas médias mínimas e médias máximas do ar e umidades relativas médias mínimas e médias máximas do ar, no interior da estufa agrícola, durante o período do experimento.

Figura 1 - Temperatura média máxima (TMAX) e média mínima (TMIN) e umidade relativa do ar média máxima (URarMAX) e média mínima (URarMIN) do ambiente físico onde foi conduzido o experimento no período de 17 de dezembro de 2016 a 28 de janeiro de 2017. UFRA/Campus Capitão Poço, 2017.

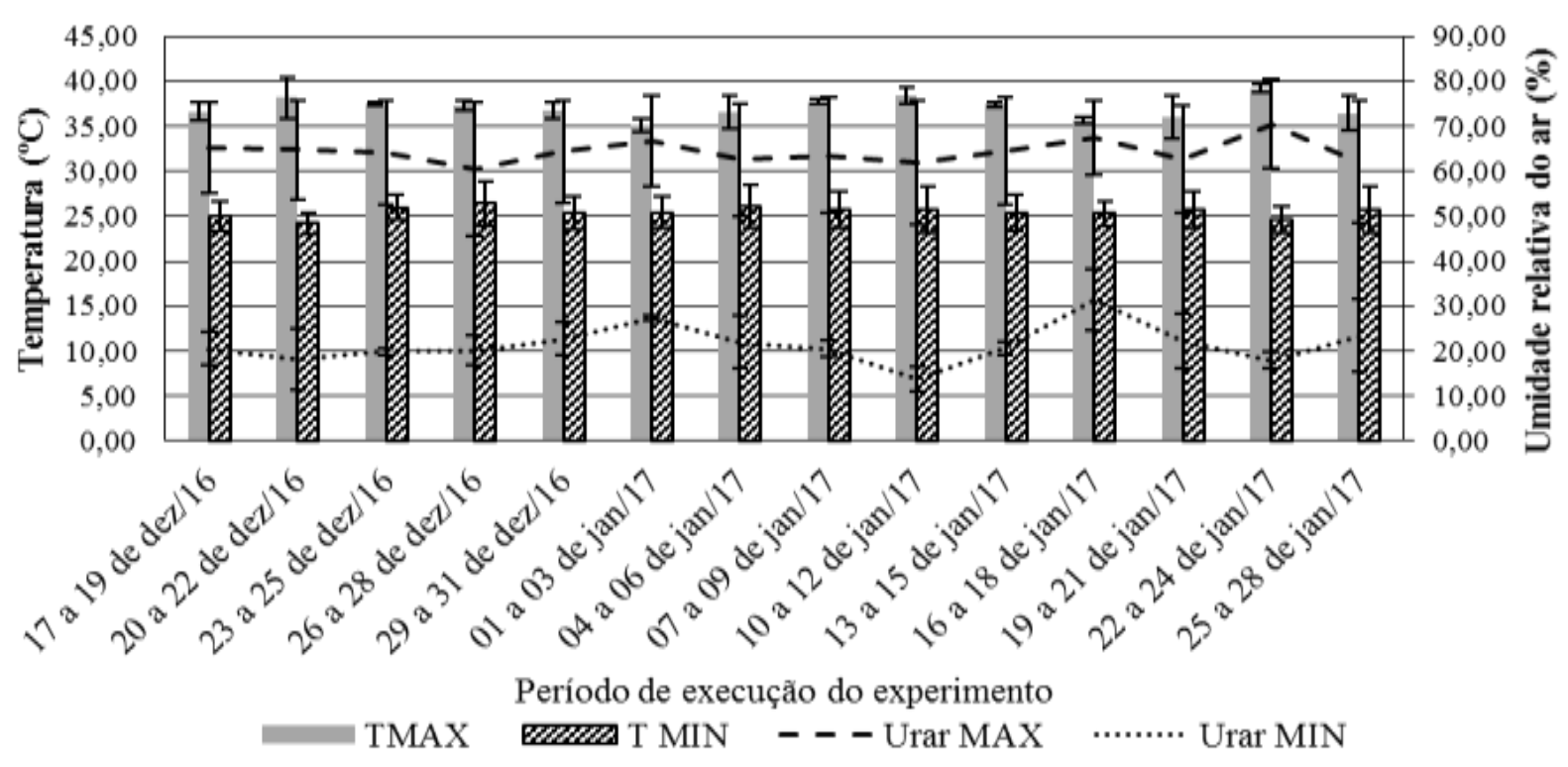

Fonte: Autores.

Foram utilizadas mudas de açaizeiro oriundas de plantas matrizes do "Programa de Melhoramento Genético do Açaizeiro", da Embrapa Amazônia Oriental, identificadas como APS. As mudas de açaizeiros foram obtidas via sementes, as quais foram semeadas em sacos de polietileno, com capacidade para $2 \mathrm{~kg}$ de um substrato, com a seguinte composição: solo local + esterco de curral curtido + pó de serragem, na proporção de 3:1:1. 
Posteriormente, essas mudas, com aproximadamente três meses de idade, foram transplantadas para vasos de polietileno preenchidos com 8,4 kg de solo (Latossolo Amarelo textura média), onde permaneceram em aclimatação por um período de três meses até o início do experimento. Antes do transplantio, o solo foi corrigido com cálcário $\left(5 \mathrm{~g} \mathrm{vaso}^{-1}\right)$ de acordo com o cálculo de necessidade de calagem baseado nos dados de análise de solo (Tabela 1) e incubado por um período de 18 dias.

Tabela 1 - Análise química da amostra do solo da camada de 0 a $20 \mathrm{~cm}$ de profundidade ${ }^{1}$. UFRA/Campus Capitão Poço, 2017.

\begin{tabular}{lccccccccc}
\hline Amostra & $\begin{array}{c}\mathrm{pH} \\
\left(\mathrm{H}_{2} \mathrm{O}\right)\end{array}$ & $\mathrm{MOS}$ & $\mathrm{CTC}$ & $\mathrm{Mg}$ & $\mathrm{Ca}$ & $\mathrm{Ca}+\mathrm{Mg}$ & $\mathrm{Al}$ & $\mathrm{P}$ & $\mathrm{K}$ \\
\cline { 2 - 8 } & & $\mathrm{g} \mathrm{kg}^{-1}$ & ----------- cmol $_{\mathrm{c}} \mathrm{dm}^{-3}$ & & & \\
\hline
\end{tabular}

Durante a fase de aclimatação as mudas foram adubadas com $3 \mathrm{~g}$ de sulfato de amônio, $3 \mathrm{~g}$ de superfosfato simples e $3 \mathrm{~g}$ de cloreto de potássio para cada vaso, e irrigadas em dias alternados. Esta operação foi realizada por meio de pesagem diária dos vasos, com o uso de uma balança com sensibilidade para 100 g. A água perdida por evapotranspiração era completada até que cada vaso atingisse $9,5 \mathrm{~kg}$, este valor foi determinado com base no dado de capacidade de campo do solo $(26 \%)$.

Em 14 de dezembro de 2016, próximo ao início da imposição dos tratamentos foi realizada uma adubação nitrogenada, aplicando-se $50 \mathrm{~mL}$ por planta de uma solução a $1 \%$ de sulfato de amônio. Os tratamentos utilizados foram: $\mathrm{T} 1=$ irrigação diária (testemunha), umidade do solo mantida entre 80 a $85 \%$ da capacidade de campo; T2 = umidade do solo reduzida por meio da suspensão da irrigação durante 3 dias e, depois, reirrigação até atingir o nível de água do tratamento controle; T3 = umidade do solo reduzida por meio da suspensão da irrigação durante 7 dias e, depois, reirrigação até atingir o nível de água do tratamento controle; T4 = umidade do solo reduzida por meio da suspensão da irrigação durante 14 dias e, depois, reirrigação até atingir o nível de água do tratamento controle; T5 = umidade do solo reduzida por meio da suspensão da irrigação durante 21 dias e, depois, reirrigação até atingir o nível de água do tratamento controle. Os tratamentos foram dispostos em delineamento inteiramente casualizado, com 4 repetições e 1 planta por parcela útil.

A manutenção dos tratamentos foi realizada através da pesagem dos vasos e pela reposição da água perdida por evapotranspiração, até atingir o peso correspondente a 9,5 kg inicialmente e após 21 dias 9,8 kg. Aos 42 dias após o inicio da aplicação dos tratamentos foram avaliadas as seguintes variáveis: a) altura das plantas (AP, cm), com o auxílio de uma trena, medida da base do caule até a maior altura da folha; b) diâmetro do caule a $3 \mathrm{~cm}$ do solo (DC, $\mathrm{cm}$ ), com o auxilio de um paquímetro; c) número de folhas (NF), por meio de contagem. Para NF foi considerado folha quando essa se apresentava com no mínimo 50\% dos folíolos completamente abertos; d) número de folíolos (Nfol), por meio de contagem; e) área foliar (Af, $\mathrm{cm} 2$ ), estimada pela relação da massa da matéria seca das folhas e massa da matéria seca de cinco discos foliares, retirados de folhas fisiologicamente maduras, obtidos com o auxílio de um perfurador de área conhecida (Benincasa, 2003; Lima; Peixoto e Ledo, 2007), utilizando-se a seguinte equação:

$$
\text { Af }=\frac{\text { PTF }}{\text { PDF }} \times \text { ACD }
$$


onde Af: área foliar, PTF: peso total das folhas (g), PDF: peso dos discos foliares (g) e ACD: área conhecida dos discos $\left(\mathrm{cm}^{2}\right)$; f) conteúdo relativo de água (CRA, em \%), obtido por meio da relação entre a diferença dos pesos das massas de matéria fresca e seca com a diferença dos pesos das matérias túrgida e seca dos discos foliares. O peso de massa de matéria túrgida foi obtido por hidratação durante 08 horas, e posterior remoção do excesso de água da superfície dos tecidos. O peso de massa de matéria seca foi obtido submetendo-se os discos em estufa de ar forçado a $60^{\circ} \mathrm{C}$ e pesando-os até peso constante. Para CRA, usou-se a equação (Barrs e Weatherley, 1962):

$$
\mathrm{CRA}=\frac{\text { PMF }- \text { PMS }}{\text { PT }- \text { PMS }}
$$

onde PMF: peso de matéria fresca dos discos foliares; PMS: peso de matéria seca dos discos foliares e PT: peso túrgido dos discos foliares.

Além disso, foram analisados: comprimento da raiz $(\mathrm{CR}, \mathrm{cm})$, medidas da base da raiz até o seu maior comprimento; volume de raiz (VR, $\mathrm{mL}$ ), com auxílio de uma proveta graduada (2000 mL); massas de matéria seca (g/planta) da raiz (MSR), caule (MSC), folha (MSF), parte aérea (MSPA= MSC + MSF), e total (MST= MSR + MSC + MSF). Para essas variáveis os materiais vegetativos foram pesados, acondicionados em sacos de papel Kraft e levados à estufa de circulação forçada de ar a $65^{\circ} \mathrm{C}$, até atingirem peso constante.

A partir dos valores da MST, bem como da Af, foram calculadas a alocação de biomassa nas folhas (ABF = MSF/MST), caule $(\mathrm{ABC}=\mathrm{MSC} / \mathrm{MST})$ e raízes $(\mathrm{ABR}=\mathrm{MSR} / \mathrm{MST})$, em $\mathrm{g} \mathrm{g}^{-1}$; a razão de área foliar (RAF = Af/MST), em $\mathrm{g} \mathrm{g}^{-}$ ${ }^{1}$ de MS; a área foliar específica (AFE = Af/MSF), em $\mathrm{g} \mathrm{g}^{-1} \mathrm{de}$ MS e a relação raiz/parte aérea (SR/PA), segundo Benincasa (2003).

A umidade do solo (US, em \%) foi obtida no primeiro dia (t0) de imposição dos tratamentos (US0) e ao final do experimento (USf), aos 42 dias (t42), adotando-se como critério avaliativo uma profundidade de 0 a $5 \mathrm{~cm}$, para coleta das amostras. Foram retiradas amostras de solo de cada repetição, mensuradas a massa úmida, em seguida as amostras foram levadas à estufa de circulação forçada de ar a $105^{\circ} \mathrm{C}$, até atingirem peso de massa constante. Para US foi utilizada a relação entre a diferença entre peso de massa úmida e peso de massa seca pelo peso de massa seca, por meio da seguinte equação:

$$
\mathrm{US}=\frac{\text { PSU-PSS }}{\text { PSS }} \times 100
$$

onde PSU é peso de amostra de solo úmido e PSS é peso de amostra de solo seco.

Os dados obtidos foram submetidos ao teste de normalidade e homocedasticidade, e à análise de variância e, quando significativos, procedeu-se a comparação das médias pelo teste de Tukey ao nível de 0,05 de probabilidade, utilizando-se o software ASSISTAT versão 7.7 beta (Silva e Azevedo, 2009).

\section{Resultados e Discussão}

Observou-se diferença significativa $(\mathrm{p}<0,05)$ nos teores de umidade do solo (USf), no final do período o experimento (t42) (Figura 2). As maiores médias de USf foram obtidas nos tratamentos, 0 e 3 dias de déficit hídrico, sem se diferenciarem significativamente entre sí. Nos demais tratamentos de níveis de déficits hídricos, os teores de USf foram reduzidos diferente e significativamente $(\mathrm{p}<0,05)$ e, apresentaram a seguinte ordem decrescente: 7; 14 e 21 dias de déficit hídrico, respectivamente (Figura 2). O teor de água disponível do solo depende em grande parte do tipo de solo. Os Latossolos Amarelos apresentam boas condições físicas de retenção de água. No entanto, em Latossolos textura média há grande percolação de água no perfil 
(EMBRAPA, 2010). Tal fato justifica os resultados para a Figura 2, em que há menor valor de umidade nos tratamentos com maior tempo de déficit hídrico, indicando que grande parte da água do solo que não foi perdida por evaporação ou evapotranspiração, sofreu percolação no perfil do solo, e apenas um baixo percentual permaneceu retido nos coloides na camada de 0 a $5 \mathrm{~cm}$ do solo.

Figura 2 - Umidade do solo no início (t0) e no final (t42) do período de imposição dos diferentes tratamentos de déficits hídricos, usados em plantas jovens de açaizeiro. Colunas com mesma letra minúscula não diferem entre si pelo teste de Tukey a 5\% de probabilidade. UFRA/Campus Capitão Poço, 2017.

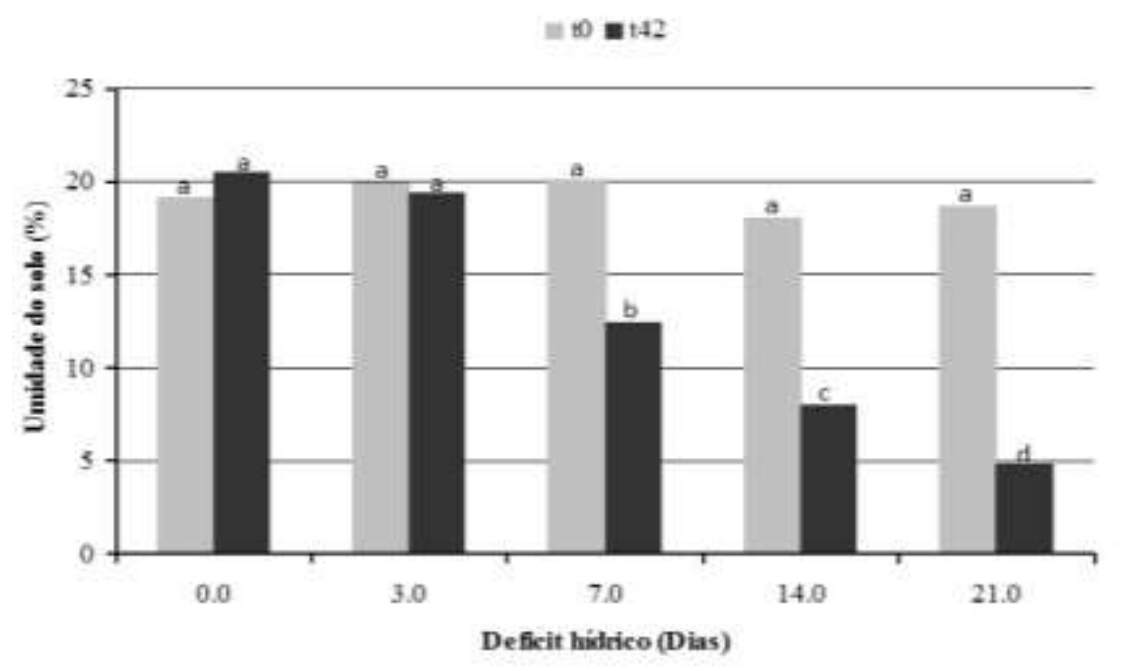

Fonte: Autores.

A AP e o NF do açaizeiro não apresentaram efeitos significativos $(\mathrm{p}<0,05)$ entre os tratamentos de déficits hídricos, entretanto, o DC dos tratamentos 14 e 21 dias, o Nfol dos tratamentos 7, 14 e 21 dias, o CR do tratamento 14 dias e o VR do tratamento 21 dias de déficits hídricos, sofreram reduções significativas ( $p<0,05)$, após 42 dias de imposição dos níveis de déficits hídricos (Tabela 2).

Tabela 2 - Altura da planta (AP), diâmetro do caule (DC), número de folhas (NF), número de folíolos (Nfol), comprimento da raiz (CR) e volume de raiz (VR), no tempo (t42) do período de imposição dos diferentes tratamentos de déficits hídricos, usados em plantas jovens de açaizeiro. UFRA/Campus Capitão Poço, 2017.

\begin{tabular}{ccccccc}
\hline $\begin{array}{c}\text { Déficit hídrico } \\
\text { (dias) }\end{array}$ & AP & DC & NF & Nfol & CR & VR \\
\cline { 2 - 7 } & ----- cm planta $^{-1}----$ & ------ un. ------- & cm planta $^{-1}$ & mL $_{\text {planta }}^{-1}$ \\
\hline 0 & $44,03 \mathrm{a}$ & $1,29 \mathrm{a}$ & $2,18 \mathrm{a}$ & $3,28 \mathrm{a}$ & $48,95 \mathrm{a}$ & $29,50 \mathrm{a}$ \\
3 & $42,13 \mathrm{a}$ & $1,12 \mathrm{ab}$ & $2,12 \mathrm{a}$ & $3,00 \mathrm{ab}$ & $45,25 \mathrm{ab}$ & $29,50 \mathrm{a}$ \\
7 & $40,25 \mathrm{a}$ & $1,08 \mathrm{ab}$ & $2,06 \mathrm{a}$ & $2,95 \mathrm{~b}$ & $46,25 \mathrm{ab}$ & $20,50 \mathrm{ab}$ \\
14 & $39,33 \mathrm{a}$ & $0,93 \mathrm{~b}$ & $1,93 \mathrm{a}$ & $2,73 \mathrm{~b}$ & $38,00 \mathrm{~b}$ & $19,75 \mathrm{ab}$ \\
21 & $39,60 \mathrm{a}$ & $0,98 \mathrm{~b}$ & $2,12 \mathrm{a}$ & $2,73 \mathrm{~b}$ & $45,75 \mathrm{ab}$ & $15,00 \mathrm{~b}$ \\
\hline CV $(\%)$ & 14,49 & 9,14 & 6,19 & 6,63 & 9,14 & 25,11 \\
\hline DMS & 13,00 & 0,22 & 0,28 & 0,43 & 8,96 & 12,54 \\
\hline
\end{tabular}

Médias seguidas pela mesma letra, na coluna, não diferem estatisticamente entre si pelo teste de Tukey ao nível de 5\% de probabilidade. Fonte: Autores. 
A redução do número de folíolos observado a partir do turno de rega aos 3 dias ocorreu como uma alternativa para reduzir a área evapotranspiratória, evitando a perda excessiva de água (Taiz et al., 2017; Kerbauy, 2019). Apesar da restrição hídrica, foi observada expansão radicular das plântulas, o que refletiu no volume de raízes. $\mathrm{O}$ alongamento do sistema radicular e diminuição da parte aérea são estratégias para a absorção de água nas camadas mais profundas do solo (Taiz et al., 2017). Entretanto, quando o estresse hídrico é severo há redução do volume de raízes em virtude da redução de fotoassimilados pela planta, como foi observado na irrigação a cada 21 dias.

Não se observou diferença estatística significativa, para MSR, entre os tratamentos com 0,3 e 7 dias de déficit hídrico, no entanto, estes foram superiores aos tratamentos com 14 e 21 dias de déficit hídrico (Tabela 3).

Tabela 3 - Massa de matéria seca da raiz (MSR), massa de matéria seca do caule (MSC), massa de matéria seca da folha (MSF), massa de matéria seca da parte aérea (MSPA), massa de matéria seca total (MST) e relação sistema radicular/parte aérea (SR/PA) no tempo (t42) do período de imposição dos diferentes tratamentos de déficits hídricos, usados em plantas jovens de açaizeiro. UFRA/Campus Capitão Poço, 2017.

\begin{tabular}{|c|c|c|c|c|c|c|}
\hline \multirow[b]{2}{*}{ Déficit hídrico (dias) } & MSR & MSC & MSF & MSPA & MST & SR/PA \\
\hline & \multicolumn{6}{|c|}{ 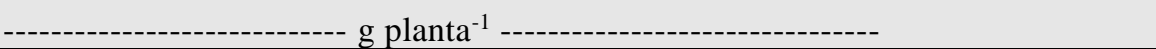 } \\
\hline 0 & $4,76 \mathrm{a}$ & $5,45 \mathrm{a}$ & $4,79 \mathrm{a}$ & $10,24 \mathrm{a}$ & $15,00 \mathrm{a}$ & $0,46 \mathrm{c}$ \\
\hline 3 & $4,09 \mathrm{a}$ & $4,13 \mathrm{~b}$ & $3,05 \mathrm{~b}$ & $7,17 \mathrm{~b}$ & $11,26 \mathrm{~b}$ & $0,57 \mathrm{bc}$ \\
\hline 7 & $4,08 \mathrm{a}$ & $2,33 c$ & $2,34 \mathrm{c}$ & $4,67 \mathrm{c}$ & $8,74 \mathrm{c}$ & $0,87 \mathrm{a}$ \\
\hline 14 & $2,71 \mathrm{~b}$ & $1,90 \mathrm{c}$ & $1,75 \mathrm{~d}$ & $3,65 \mathrm{~d}$ & $6,35 \mathrm{~d}$ & $0,74 \mathrm{ab}$ \\
\hline 21 & $2,28 \mathrm{~b}$ & $1,80 \mathrm{c}$ & $1,74 \mathrm{~d}$ & $3,54 \mathrm{~d}$ & $5,81 \mathrm{~d}$ & $0,64 \mathrm{bc}$ \\
\hline $\mathrm{CV}(\%)$ & 12,65 & 8,32 & 6,64 & 5,85 & 9,17 & 13,32 \\
\hline DMS & 0,99 & 0,57 & 0,40 & 0,75 & 1,89 & 0,19 \\
\hline
\end{tabular}

Médias seguidas pela mesma letra, na coluna, não diferem estatisticamente entre si pelo teste de Tukey ao nível de 5\% de probabilidade. Fonte: Autores.

O tratamento controle, com média de 5,45, 4,79, 10,24 e 15,00 g/planta, nesta ordem para massa de matéria seca do caule, folha, parte aérea e total, mostrou-se superior a todos os tratamentos com níveis de déficit hídrico (Tabela 3). Foram verificadas reduções em relação à testemunha, nesta ordem, para 3, 7, 14 e 21 dias de déficit hídrico, 19,89\%, 57,25\%, 65,14\%, 66,97\%, para MSC; 36,33\%; 51,15\%; 63,47\%; 63,67\%, para MSF; 29,98\%, 54,39\%, 64,36\%, 65,43\%, para MSPA; 24,93\%, 41,73\%, 57,67\%, 61,27\%, para MST (Tabela 3). Mar et al. (2013) observaram que a produção de biomassa das diferentes partes da planta jovens de açaizeiro foi fortemente influenciada pelo déficit hídrico. A produção de biomassa seca no final do experimento (44 dias) foi comprometida nas plantas sob déficit hídrico, observando-se reduções de 38, 41, 37 e $38 \%$ na biomassa seca de folha, raiz, caule e total, respectivamente, quando comparadas às plantas controle.

A maior relação SR/PA foi observada aos sete dias de déficit hídrico, no entanto, este não diferiu estatisticamente com o de 21 e 14 dias de déficit hídrico (Tabela 3). Estes resultados, corroboram os resultados apresentados para MSPA (Tabela 3), em que esses tratamentos com 7 e 14 e 21 dias de déficit hídrico apresentaram menores valores, indicando que as plantas submetidas à maiores déficits hídricos, tendem a distribuir mais biomassa para as raízes, como medida de sobrevivência frente ao déficit hídrico (Silvestre et al., 2017; Kerbauy, 2019).

Essas reduções de produtividade primária das plantas jovens de açaizeiros sob déficit hídrico, representadas pelas produções de MSF, MSAC, MSR, MSPA, MST e Af são devidas principalmente às reduções da umidade do solo (Mar et al., 2013). Silveira et al. (2016) ao estudarem o efeito de regimes hídricos em mudas de açaizeiro, observaram efeito negativo dos 
tratamentos com umidade do solo abaixo de $70 \%$ da capacidade de campo nas variáveis biométricas e produção de matéria seca, fato em decorrência da diminuição da taxa transpiratória e atividade fotossintética.

Na Tabela 4, observa-se diferença estatística significativa para alocação de biomassa na raiz, caule e folha, entre os diferentes tratamentos. Para alocação de biomassa na raiz, verificou-se maior alocação de biomassa para os tratamentos com maiores déficits hídricos, respectivamente, para 7, 14 e 21 dias de déficit hídrico (Tabela 4). Estes resultados podem ser explicados pelo maior comprimento de raízes apresentados, em busca da maior absorção de água por este órgão. Silveira et al. (2017) também observaram aumento do sistema radicular em mudas de açaizeiro sob condição de restrição hídrica.

Para alocação de biomassa no caule as maiores médias foram obtidas nos tratamentos com 0 e 3 dias de déficit hídrico, porém não diferiram estatisticamente do tratamento com 21 dias de déficit hídrico (Tabela 4). Para alocação de biomassa na folha, as maiores médias foram verificadas na testemunha, no entanto não diferiu, estatisticamente, das médias observadas para o tratamento com 21 dias de déficit hídrico (Tabela 4). Estes resultados, para ABC e ABF, corroboram os resultados observados para $\mathrm{ABR}$, sugerindo-se maior alocação de biomassa para as raízes em tratamentos com maior déficit hídrico e maior alocação de biomassa para a parte aérea nos tratamentos com menor déficit hídrico. Para justificar os valores para $\mathrm{ABR}, \mathrm{ABC}$ e $\mathrm{ABF}$, com 21 dias de déficit hídrico, sugere-se, que para estas plantas, o alto grau de estresse reduziu a alocação de biomassa tanto para as raízes quanto para a parte aérea. Taiz et al., (2017) explicam que a redução da parte área é uma alternativa para evitar a perda de água por evapotranspiração.

Tabela 4 - Alocação de biomassa nas raízes (ABR), alocação de biomassa no caule (ABC) e alocação de biomassa nas folhas ( $\mathrm{ABF}$ ) no tempo (t42) do período de imposição dos diferentes tratamentos de déficits hídricos, usados em plantas jovens de açaizeiro. UFRA/Campus Capitão Poço, 2017.

\begin{tabular}{|c|c|c|c|}
\hline \multirow[b]{2}{*}{ Déficit hídrico (dias) } & $\mathrm{ABR}$ & $\mathrm{ABC}$ & $\mathrm{ABF}$ \\
\hline & \multicolumn{3}{|c|}{ 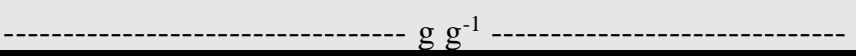 } \\
\hline 0 & $0,31 \mathrm{c}$ & $0,37 \mathrm{a}$ & $0,32 \mathrm{a}$ \\
\hline 3 & $0,36 \mathrm{bc}$ & $0,37 \mathrm{a}$ & $0,27 \mathrm{~b}$ \\
\hline 7 & $0,46 \mathrm{a}$ & $0,27 \mathrm{~b}$ & $0,27 \mathrm{~b}$ \\
\hline 14 & $0,43 \mathrm{ab}$ & $0,30 \mathrm{~b}$ & $0,28 \mathrm{~b}$ \\
\hline 21 & $0,39 \mathrm{abc}$ & $0,31 \mathrm{ab}$ & $0,30 \mathrm{ab}$ \\
\hline $\mathrm{CV}(\%)$ & 10,47 & 9,37 & 6,01 \\
\hline DMS & 0,09 & 0,07 & 0,04 \\
\hline
\end{tabular}

Médias seguidas pela mesma letra, na coluna, não diferem estatisticamente entre si pelo teste de Tukey ao nível de 5\% de probabilidade. Fonte: Autores.

Todos os tratamentos com níveis de déficits hídricos apresentaram-se inferiores a testemunha para as variáveis Af e AfP (Tabela 5). Estes resultados estão de acordo com Mar et al. (2013), a área foliar de plantas jovens de açaizeiros submetidas a ciclos de deficiência hídrica foi reduzida.

A redução da área foliar nas plantas ocorre pela redução da divisão e expansão celular, alteração na morfologia foliar e iniciação da senescência e abscisão foliar. Essa redução resulta em menor transpiração e maior conservação do suprimento de água no solo ao longo do tempo, embora limite a produção fotossintética da planta (Taiz et al., 2017). 
Tabela 5 - Área foliar (Af), área foliar por planta (AfP), área foliar específica (AfE), razão de área foliar (RAF) no tempo (t42) do período de imposição dos diferentes tratamentos de déficits hídricos, usados em plantas jovens de açaizeiro. UFRA/Campus Capitão Poço, 2017.

\begin{tabular}{ccccc}
\hline & & Af & AfE & RAF \\
\cline { 4 - 5 } Déficit hídrico (dias) & $\begin{array}{c}\text { AfP } \\
\mathrm{cm}^{2} \text { folha }^{-1}\end{array}$ & $\mathrm{~cm}^{2}$ planta $^{-1}$ & -------- $\mathrm{g} \mathrm{g}^{1}$ de MS -------- \\
\hline 0 & $201,77 \mathrm{a}$ & $951,80 \mathrm{a}$ & $198,88 \mathrm{~b}$ & $63,72 \mathrm{a}$ \\
3 & $134,21 \mathrm{~b}$ & $631,53 \mathrm{~b}$ & $207,27 \mathrm{a}$ & $56,14 \mathrm{ab}$ \\
7 & $105,80 \mathrm{bc}$ & $461,82 \mathrm{c}$ & $197,27 \mathrm{~b}$ & $53,02 \mathrm{~b}$ \\
14 & $81,96 \mathrm{c}$ & $363,76 \mathrm{~d}$ & $207,63 \mathrm{a}$ & $57,28 \mathrm{ab}$ \\
21 & $78,17 \mathrm{c}$ & $346,36 \mathrm{~d}$ & $199,20 \mathrm{~b}$ & $59,66 \mathrm{ab}$ \\
\hline CV $(\%)$ & 12,69 & 6,60 & 3,77 & 6,04 \\
\hline DMS & 33,39 & 79,51 & 3,39 & 7,64 \\
\hline
\end{tabular}

Médias seguidas pela mesma letra, na coluna, não diferem estatisticamente entre si pelo teste de Tukey ao nível de 5\% de probabilidade. Fonte: Autores.

A área foliar específica (AfE) expressa a razão entre área foliar e massa seca da folha. Os tratamentos 3 dias e 14 dias de déficit hídrico apresentaram valores superiores quando comparados aos demais tratamentos, inclusive à testemunha, quando se trata de AfE (Tabela 5) com médias de 207,27 e 207,63 $\mathrm{cm}^{2} \mathrm{~g}^{-1}$, respectivamente.

A razão de área foliar $\left(\mathrm{cm}^{2} \mathrm{~g}^{-1}\right)$ expressa à área foliar útil para fotossíntese (Benincasa, 2003). Ao tratar do parâmetro RAF (tabela 5), é possível dizer que, com exceção do tratamento que teve um déficit hídrico de 7 dias, apresentando comportamento inferior em relação a testemunha, os demais tratamentos não diferiram estatisticamente do tratamento controle, irrigado diariamente.

Foram observadas reduções no CRA de 3,19\%, 3,98\%, 10,13\%, 12,39\%, respectivamente, para os tratamentos com 3, 7, 14 e 21 dias de déficit hídrico (Figura 4). A redução do conteúdo relativo de água em condições de estresse hídrico é decorrente da diminuição da abertura estomática e, consequentemente, da transpiração das mudas. Esse comportamento foi verificado em estudos anteriores com palmeiras em condições de estresse hídrico (Silva et al., 2016; Silvestre et al, 2016; 2017). Calbo e Moraes (2000) observaram que a suspensão da irrigação após 24 dias reduziu drasticamente a transpiração e condutância estomática das mudas de açaizeiro. 
Figura 4 - Conteúdo relativo de água no final (t42) do período de imposição dos diferentes tratamentos de déficits hídricos, usados em plantas jovens de açaizeiro. Colunas com mesma letra minúscula não diferem entre si pelo teste de Tukey a 5\% de probabilidade. UFRA/Campus Capitão Poço, 2017.

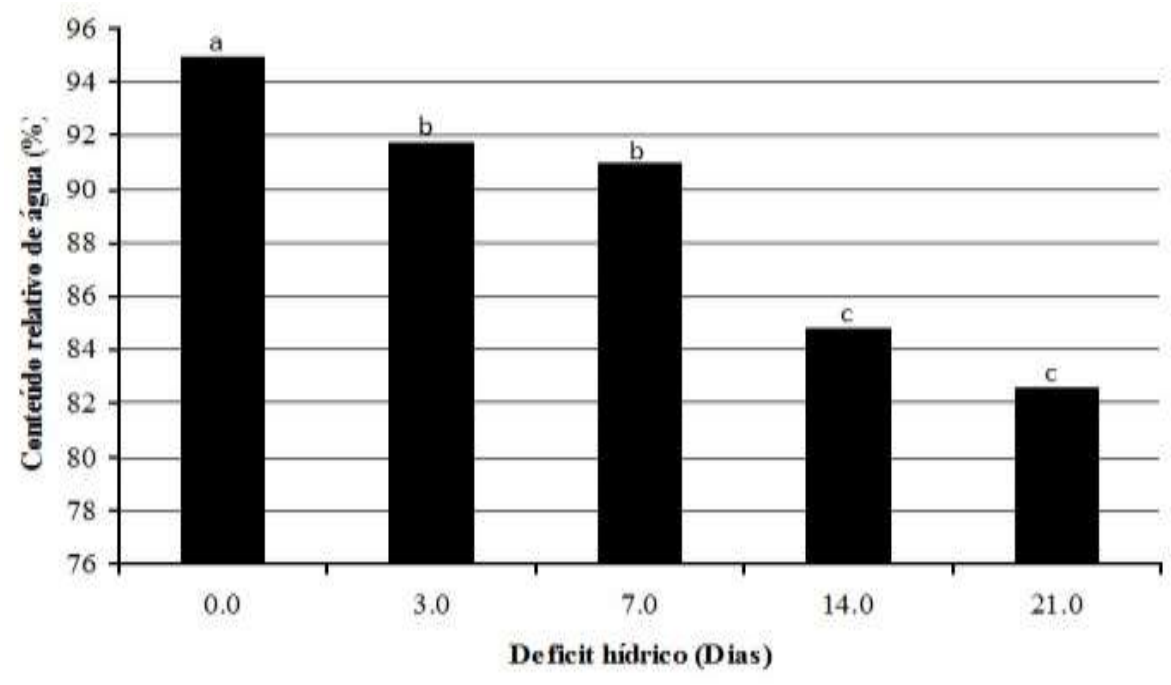

Fonte: Autores.

\section{Conclusão}

O déficit hídrico influenciou negativamente o desenvolvimento vegetativo de plantas jovens de açaizeiro.

O Estresse hídrico a partir de três dias promoveu reduções significativas no conteúdo relativo de água, área foliar e nas produções de massas secas, com exceção da massa seca da raiz, em plantas jovens de açaizeiro.

\section{Referências}

Araújo, C. S., Rufino, C. P. B., Bezerra, J. L. S., Andrade Neto, R. C., \& Lunz, A. M. P. (2018). Crescimento de mudas de açaizeiro (Euterpe oleracea Mart.) submetidas a diferentes doses de fósforo. South American Journal of Basic Education, Technical and Technological, 5(1), $102-111$.

Barrs, H. D., \& Weatherley, P. E. (1962). A re-examination of the relative turgidity technique for estimating water deficits in leaves. Australian journal of biological sciences, 15(3), 413-428.

Benincasa, M. M. P. (2003). Análise de crescimento de plantas (noções básicas). FUNEP.

Calbo, M. E. R., \& Moraes, J. A. P. D. (2000). Efeitos da deficiência de água em plantas de Euterpe oleracea (açaí). Brazilian Journal of Botany, 23, 225-230.

Cordeiro, Y. E. M., Tavares, F. B., Nascimento, A. W. S., \& Pena, H. W. A. (2017). Aspectos bioquímicos de plantas jovens de açaízeiro (Euterpe oleraceae) sob dois regimes hídricos na Amazônia Oriental. Biota Amazônia, 7(3), 52-56. http://doi.org/10.18561/2179-5746/biotaamazonia.v7n3p52-56

Empresa Brasileira de Pesquisa Agropecuária - EMBRAPA. (2010). Latossolos amarelos. Disponível em <http://www.agencia.cnptia.embrapa.br>. Acesso em 09 de fevereiro de 2017.

Empresa Brasileira de Pesquisa Agropecuária - EMBRAPA. (2013). Sistema brasileiro de classificação de solos.

Homma, A. K. O., Nogueira, O. L., Menezes, A. J. E. A., Carvalho, J. E. U., Nicoli, C. M. L., \& Matos, G. B. (2006). Açaí: novos desafios e tendências. Amazônia: Ciência \& Desenvolvimento, 1(2), 7-23.

IBGE. (2021). Pesquisa Agrícola Municipal. https://sidra.ibge.gov.br/pesquisa/pam/tabelas.

Kerbauy, G. B. (2019). Fisiologia vegetal. Guanabara Koogan.

Lima, J. F., Peixoto, C. P., \& Ledo, C. A. S. (2007). Índices fisiológicos e crescimento inicial de mamoeiro (Carica papaya L.) em casa de vegetação. Ciência e Agrotecnologia, 31, 1358-1363. https://doi.org/10.1590/S1413-70542007000500013

Mar, C.C., Conceição, H. E. O., Santos, A. B. R., Viégas, I. J. M., \& Silva, F. S. N. (2014). Produção de massa seca e área foliar do açaizeiro sob déficit hídrico. Revista Agroecossistemas, 5(2), 14-23. http://doi.org/10.18542/ragros.v5i2.1794 
Research, Society and Development, v. 10, n. 12, e496101220582, 2021

(CC BY 4.0) | ISSN 2525-3409 | DOI: http://dx.doi.org/10.33448/rsd-v10i12.20582

Silva, P. A., Oliveira, I. V., Rodrigues, K. C. B., Cosme, V. S., Bastos, A. J. R., Detmann, K. S. C., Cunha, R. L., Festucci-Buselli, R. A., DaMatta, F. M., \& Pinheiro, H. A. (2016) Leaf gas exchange and multiple enzymatic and non-enzymatic antioxidant strategies related to drought tolerance in two oil palm hybrids. Trees, 30:203-214. https://doi.org/10.1007/s00468-015-1289-x

Silva, A. C. D., Smiderle, O. J., Oliveira, J. M. F., \& Silva, T. J. (2017). Tamanho da semente e substratos na produção de mudas de açaí. Advances in Forestry Science, 4(4), 151-156. https://doi.org/10.34062/afs.v4i4.4590

Silva, F. A. S., \& Azevedo, C. A. V. (2009). Principal Components Analysis in the Software Assistat-Statistical Attendance. In: World Congress On Computers In Agriculture, 7, Reno-NV-USA: American Society of Agricultural and Biological Engineers.

Silvestre, W. V. D., Silva, P. A., Palheta, L. F., Oliveira Neto, C. F., Souza, R. O. R. M., Festucci-Buselli, R. A., \& Pinheiro, H. A. (2017). Differential tolerance to water deficit in two açaí (Euterpe oleracea Mart.) plant materials. Acta physiologiae plantarum, 39(4), 1-10. https://doi.org/10.1007/s11738-016$2301-9$

Silvestre, W. V. D., Pinheiro, H. A., Souza, R. O. M., \& Palheta, L. F. (2016). Morphological and physiological responses of açaí seedlings subjected to different watering regimes. Revista Brasileira de Engenharia Agrícola e Ambiental, 20(4), 364-371. https://doi.org/10.1590/1807-1929/agriambi.v20n4p364371

Taiz, L. Zeiger, E. MØler, I. M. \& Murphy, A. (2017). Fisiologia e desenvolvimento vegetal. Artmed. 